Tohoku J. Exp. Med., 2012, 226, 19-27

\title{
Association between Increased Serum Osteoprotegerin Levels and Improvement in Bone Mineral Density after Parathyroidectomy in Hemodialysis Patients
}

\author{
Cai-Mei Zheng, ${ }^{1, *}$ Pauling Chu, ${ }^{2, *}$ Chia-Chao Wu, ${ }^{2}$ Wen-Ya Ma, Kuo-Chin Hung, ${ }^{3}$ \\ Yung-Ho Hsu, ${ }^{1}$ Yuh-Feng Lin, ${ }^{1}$ Liang-Kuang Diang ${ }^{2}$ and Kuo-Cheng $\mathrm{Lu}^{3}$ \\ ${ }^{1}$ Division of Nephrology, Department of Medicine, Taipei Medical University-Shuang Ho Hospital, Taipei
Medical University, Taipei, Taiwan
${ }^{2}$ Division of Nephrology, Department of Medicine, Tri-Service General Hospital, National Defense Medical
Center, Taipei, Taiwan
${ }^{3}$ Department of Medicine, Cardinal Tien Hospital, School of Medicine, Fu-Jen Catholic University, New Taipei
City, Taiwan
}

Secondary hyperparathyroidism (SHPT) is a common complication in chronic renal disease. Osteoprotegerin (OPG), an extracellular cytokine receptor secreted by osteoblasts, can promote bone formation by inhibiting the function of osteoclasts. Hemodialysis (HD) patients have elevated serum OPG levels. OPG secretion can be suppressed with high parathyroid hormone (PTH) levels. HD patients with refractory SHPT can benefit from parathyroidectomy (PTX) treatment, but the changes of serum OPG, bone turnover markers and bone mineral density (BMD) following PTX in HD patients remain unclear. In this study, patients on maintenance HD who received PTX for refractory SHPT $(n=28)$ were prospectively followed for 1 year. Serum intact PTH (iPTH), alkaline phosphatase (Alk-P), and OPG were measured serially; BMD was measured pre-PTX and at 1 year after PTX. After PTX, serum iPTH levels reduced profoundly. Serum Alk-P levels increased rapidly, peaking at 2 weeks post-PTX, while serum OPG levels gradually increased at 2 weeks after PTX and peaked at 2 months. BMD improved in both femoral neck (FN; cancellous and cortical bone) and lumbar spine (LS; cancellous bone). Higher baseline iPTH levels were associated with greater FN and LS BMD improvements at one year after PTX. The increment of serum OPG was correlated with the increase in LS BMD, implying that inhibition of osteoclastic bone resorption may improve BMD within the first year after PTX. These findings suggest that PTX removes the suppressive effects of high PTH on OPG secretion, resulting in the increased serum OPG levels that may contribute to BMD improvement.

Keywords: alkaline phosphatase; bone mineral density; hemodialysis; osteoprotegerin; parathyroidectomy Tohoku J. Exp. Med., 2012, 226 (1), 19-27. ㅇ 2012 Tohoku University Medical Press

Secondary hyperparathyroidism (SHPT), a common complication in chronic renal disease, results from vitamin $\mathrm{D}$ deficiency, impaired mineral metabolism, and decreased renal function. SHPT is responsible for various disorders, including renal osteodystrophy (ROD), extraskeletal calcification, and cardiovascular disease (CVD), each of which increases the morbidity and mortality of these patients (Melamed et al. 2008; Tentori et al. 2008; Floege et al. 2011). Elevated levels of parathyroid hormone (PTH) stimulate bone demineralization and lead to high bone turnover, a condition characterized by discordance between the formation and resorption of bone, with resorption being prominent. In medically refractory SHPT, the new bone formed is structurally inferior and fragile, with an increased fracture risk. The typical histological form of this condition is called either osteitis fibrosis or mixed osteodystrophy (Malluche and Faugere 1990; Sherrard et al. 1993).

Hemodialysis (HD) patients exhibit higher than normal levels of osteoprotegerin (OPG), a decoy receptor that influences the PTH-bone metabolism pathway through its inhibitory action on osteoclasts. OPG may also promote bone regeneration and enhance new bone formation (Yao et al. 2011). Dialysis patients exhibit higher serum OPG levels and lower serum levels of receptor activator of nuclear factor kappa-B ligand (RANKL), most likely to compensate for higher osteoclast activity. Serum OPG levels are lower

Received October 6, 2011; revision accepted for publication November 26, 2011. doi: 10.1620/tjem.226.19

*These authors have equally contributed to this work.

Correspondence: Kuo-Cheng Lu, Division of Nephrology, Department of Medicine, Cardinal Tien Hospital, School of Medicine, Fu-Jen Catholic University, 362, Chung-Cheng Rd, Hsin-Tien District, New Taipei City, Taiwan.

e-mail: kuochenglu@gmail.com 
in patients with adynamic bone disease (ABD) than in patients with predominant SHPT (Coen et al. 2002). Approximately $50 \%$ of peritoneal dialysis patients show elevated OPG levels, indicating disturbed osteoclast function (Grzegorzewska and Mlot 2005). HD patients with low femoral neck bone mineral density (BMD) display higher OPG levels than patients with normal BMD (Doumouchtsis et al. 2008).

Parathyroidectomy (PTX) is a frequently used therapeutic procedure for managing refractory SHPT; this surgery has a profound effect on recovery of BMD (Stilgren et al. 2004). A recent histomorphological study found that BMD increased after PTX due to abundant secondary mineralization by osteocytes, with diffuse mineralization observed in both the basic multicellular unit and the bone structural unit (Yajima et al. 2010). Since OPG suppresses bone resorption and increased alkaline phosphatase (Alk-P) activity is associated with bone formation, both of these markers may be elevated during bone anabolism. High serum levels of Alk-P and OPG were reported in a study of high turnover bone disease (Yano et al. 1999). However, the nature of the relationship between serum OPG and bone turnover markers following PTX is still debated.

The current study evaluated serial changes in iPTH, Alk-P, and OPG levels after PTX in dialysis patients, with the aim of clarifying the relationship between these markers and BMD changes during the first year after PTX. Our results demonstrated an association among OPG, Alk-P, and BMD changes.

\section{Material and Methods}

\section{Patients}

The study sample comprised 30 patients with refractory SHPT who were receiving long-term hemodialysis therapy. In this study, PTX was considered necessary when, despite optimal medical and dietary treatment, high serum iPTH persisted in combination with hypercalcemia, vascular calcification, severe osteopathy, drug-resistant hyperphosphatemia, and calciphylaxis. In addition, the diagnostic process was required to exclude adynamic bone disease (Frazao and Martins 2009). All 30 patients underwent PTX in the nephrology unit of either the Cardinal Tien Hospital or the Tri-Service General Hospital, both situated in Taipei, Taiwan.

In patients with recurrent SHPT, the iPTH level normalizes $(<60 \mathrm{pg} / \mathrm{ml})$ within 2 weeks after PTX, but becomes elevated again more than 6 months postoperatively (Yatsuka et al. 2002; Chen et al. 2011). Patients with SHPT were excluded from the current study. This meant that data from 2 patients $(6.7 \%$ of the sample or $2 / 30)$ were excluded from analysis because of recurrent SHPT during the 12-month followup. Additional potential participants had been excluded from the study because of the presence of recent infections, gastrointestinal diseases, chronic alcoholism, malignant diseases, coronary artery disease, and chronic obstructive pulmonary disease; or if the patient used mineralocorticoids, immunosuppressants, or anabolic agents (Epstein et al. 2001; Caplan et al. 2011). Patients who had received renal transplants were also excluded.

The final sample of 28 patients included 9 pre-menopausal women, 5 post-menopausal women, and 14 men; the overall sample's mean age was $49.5 \pm 10.5$ years. The mean duration of hemodialysis therapy was $66.5 \pm 16.4$ months. The causes of renal failure were diabetic nephropathy $(n=6)$, hypertensive nephrosclerosis $(n=3)$, chronic glomerulonephritis $(n=9)$, polycystic kidney disease $(n=1)$, analgesic nephropathy $(n=1)$, and unknown causes $(n=8)$. No study patients had received aluminum-containing phosphate binders for at least 1 year prior to surgery. All patients had serum aluminum levels $<1.0 \mu \mathrm{mol} / \mathrm{l}$. Each patient's body mass index (BMI) was calculated as body weight divided by the square of the body height $\left(\mathrm{kg} / \mathrm{m}^{2}\right)$. The normal range for BMI is $18.5 \mathrm{~kg} / \mathrm{m}^{2}$ to $24 \mathrm{~kg} / \mathrm{m}^{2}$.

The control group (serum iPTH level $<300 \mathrm{pg} / \mathrm{ml}$ ) consisted of 30 patients on long-term hemodialysis, whose sex and age distributions were similar to those of the study patients. One patient in the control group was lost during followup, and 1 patient died of sepsis; thus, the data of only 28 control patients were included in the final analysis (hence, the same number as the study group). The study was approved by the Institutional Review Board of Cardinal Tien Hospital and Tri-Service General Hospital (Taipei, Taiwan).

\section{Parathyroidectomy (PTX)}

Preoperative imaging was not performed routinely because four gland surgery was planned with Sestamibi scanning rarely showing all 4 glands (Coulston et al. 2010). Those who had less than 4 glands removed received a thymectomy, which revealed no further parathyroid glands. The mean number of removed parathyroid glands was $3.93 \pm 0.26$ per patient. After total PTX had been performed, a small piece of the resected parathyroid tissue was divided into approximately 1-mm diameter pieces, and was autotransplanted into the subcutaneous fat of 1 forearm. Approximately $100 \mathrm{mg}$ of tissue was grafted into each patient. Although vitamin D supplements had not been administered to the patients for at least 1 month before surgery, postoperatively they all received oral treatment with calcitriol $\left(1,25(\mathrm{OH})_{2}\right.$ Vit $\left.\mathrm{D}_{3}, 2.0 \mu \mathrm{g} / \mathrm{d}\right)$. Calcitriol was administered orally postsurgery for 3 weeks after PTX to counteract the severe impairment in bone mineralization caused by decreased serum phosphorus and calcium levels (Yajima et al. 2003). In addition, intravenous (i.v.) calcium gluconate was administered after surgery when required. Some patients also received calcium supplements at doses established daily.

In all patients, the iPTH level was $<100 \mathrm{pg} / \mathrm{ml}$ postoperatively, and the serum calcium level was maintained at approximately 8 to 9 $\mathrm{mg} / \mathrm{dl}$. Thereafter, in cases where the iPTH level rose to $>100 \mathrm{pg} / \mathrm{ml}$, the serum calcium level was maintained at roughly 9 to $10 \mathrm{mg} / \mathrm{dl}$ by adding calcium salts and low-dose active vitamin $\mathrm{D}$ to prevent recurrent HPT. The vitamin D was discontinued when iPTH fell to $<70$ $\mathrm{pg} / \mathrm{ml}$, to avoid adynamic bone disease (Yajima et al. 2001).

\section{Blood Measurements \\ Analysis of serum biochemical and bone metabolism parameters}

On the day of PTX, after patients had fasted for $10 \mathrm{~h}$, blood was drawn at 8:00 am and 9:00 am, and centrifuged within $30 \mathrm{~min}$; this yielded the baseline (Day 0 or D0) level before the operation. After PTX, further blood samples were drawn on Day 2 (D2), and at 1 week (W1), 2 weeks (W2), 1 month (M1), 2 months (M2), 3 months (M3), 6 months (M6), 9 months (M9), and 12 months (M12). After collection, serum samples were immediately frozen and stored at $-70^{\circ} \mathrm{C}$ until use. Samples were used to determine the levels of iPTH, phosphate, calcium, and other bone metabolism parameters. Total calcium, serum phosphate, Alk-P, and serum albumin were determined with an AU5000 automated chemistry analyzer (Olympus, 
Tokyo, Japan). Serum iPTH levels were measured in an immunoradiometric assay (Nichols Institute Diagnostics, San Juan Capistrano, CA, USA). Serum aluminum levels were measured with atomic absorption spectrometry in a graphite oven. Serum OPG (RayBiotech Inc., Norcross, GA) levels were measured using a commercially available enzyme-linked immunosorbent assay (ELISA).

\section{BMD measurements}

All patients underwent dual-energy X-ray absorptiometry (DEXA) scans to determine the BMD of the LS (L2-L4; cancellous bone) and the left FN (cancellous and cortical bone). A single technician certified by the International Society of Clinical Densitometry performed all DEXA scans on a fan-beam bone densitometer (QDR4500A; Hologic, Waltham, MA, USA) (Huang et al. 2009). DEXA data for age- and sex-matched controls were obtained from the DEXA manufacturer's reference population, and comprised the BMD reference in this study.

Because BMD is influenced by age, sex, and renal osteodystrophy, previous research has recommended using T-scores to measure BMD in dialysis patients (Miller 2009). In this study, BMD was expressed as the T-score.

To evaluate the possible effects of aortic calcification on LS BMD assessment, lateral abdominal radiograph, which included vertebrae from at least T11 to L5, was recorded in most of our patients before and 1 year after PTX. None of the patients displayed extensive aortic calcification.

\section{Statistical analysis}

All data are reported as means and standard deviations (SD), unless otherwise stated. All statistical tests were performed with the Statistical Package for Social Sciences (SPSS version 17.0) for Windows (SPSS Inc, Chicago, IL). Univariate simple regression analysis was used to assess the linear relationship between parameters. The Shapiro-Wilk test of normality was used to test the distribution of variables, and the Spearman correlation coefficients were calculated when the normality test failed. A $P$-value of $<0.05$ was considered statistically significant.

\section{Results}

\section{Participant characteristics}

General characteristics, clinical variables, and biochemical parameters of the study participants are shown in Table 1. Compared with the control group, patients with SHPT had higher baseline (pre-PTX) levels of iPTH (normal value [NV] 10-65 pg/ml), Alk-P (NV 60-170 IU/1), OPG (NV 259.7 $\pm 65.2 \mathrm{pg} / \mathrm{ml}$ ), total calcium (NV 8.5-10.5 $\mathrm{mg} / \mathrm{dl}$ ), and phosphate (NV 2.3-4.6 mg/dl).

We separated the study participants into 3 subgroups for analysis, namely pre-menopausal women $(n=9)$, postmenopausal women $(n=5)$, and men $(n=14)$. For the baseline OPG level, we found no significant difference between post-menopausal women and the men $(1,568.72 \pm$ 541.6 vs $2,238.0 \pm 782.2$, respectively; $P=.098$ ). However,

Table 1. Comparison between clinical variables and biochemical parameters before and one year after parathyroidectomy (PTX).

\begin{tabular}{|c|c|c|c|c|}
\hline & \multicolumn{2}{|c|}{$\operatorname{PTX}(n=28)$} & \multicolumn{2}{|c|}{ Control $(n=28)$} \\
\hline Age, years & \multicolumn{2}{|c|}{$49.5 \pm 10.5$} & \multicolumn{2}{|c|}{$49.8 \pm 11.8$} \\
\hline $\operatorname{Sex}(M / F)$ & \multicolumn{2}{|c|}{$14 / 14$} & \multicolumn{2}{|c|}{$14 / 14$} \\
\hline \multirow[t]{2}{*}{ HD duration } & \multicolumn{2}{|c|}{$66.5 \pm 16.4$ months } & \multicolumn{2}{|c|}{$68.5 \pm 18.2$ months } \\
\hline & D0 & M12 & D0 & M12 \\
\hline iPTH, pg/ml & $1,263.8 \pm 543.0^{\mathrm{b}}$ & $48.1 \pm 19.5^{\mathrm{d}}$ & $187.9 \pm 75.2^{\mathrm{f}}$ & $194.4 \pm 89.6^{\mathrm{f}}$ \\
\hline Alk-P, IU/1 & $610.3 \pm 258.0^{\mathrm{b}}$ & $284.0 \pm 76.0^{\mathrm{d}}$ & $108.2 \pm 19.7^{\mathrm{f}}$ & $116.8 \pm 22.7^{\mathrm{f}}$ \\
\hline $\mathrm{OPG}, \mathrm{pg} / \mathrm{ml}$ & $2,167.1 \pm 767.8^{b}$ & $2,226.0 \pm 741.5^{\mathrm{d}}$ & $1,576.4 \pm 268.4^{\mathrm{f}}$ & $1,624.3 \pm 312.7^{\mathrm{f}}$ \\
\hline FN T-score & $-0.94 \pm 0.59^{\mathrm{a}}$ & $-0.39 \pm 0.51^{\mathrm{d}}$ & $-0.41 \pm 0.55$ & $-0.45 \pm 0.66$ \\
\hline FN BMD $\mathrm{g} / \mathrm{cm}^{2}$ & $0.72 \pm 0.07^{\mathrm{a}}$ & $0.78 \pm 0.06^{\mathrm{d}}$ & $0.79 \pm 0.08$ & $0.78 \pm 0.09$ \\
\hline FN FC BMD & & $+8.84 \pm 3.43 \%$ & & $-1.26 \pm 0.38 \%$ \\
\hline LS T-score & $-0.28 \pm 0.52^{\mathrm{a}}$ & $0.76 \pm 0.50^{\mathrm{d}}$ & $0.47 \pm 0.46^{\mathrm{e}}$ & $0.27 \pm 0.48^{\mathrm{f}}$ \\
\hline LS BMD g/ $/ \mathrm{cm}^{2}$ & $0.97 \pm 0.07^{\mathrm{a}}$ & $1.09 \pm 0.07^{\mathrm{d}}$ & $1.02 \pm 0.08^{\mathrm{f}}$ & $1.03 \pm 0.09^{\mathrm{f}}$ \\
\hline LS FC BMD & & $+12.50 \pm 3.86 \%$ & & $+0.98 \pm 0.29 \%$ \\
\hline $\mathrm{TCa}, \mathrm{mg} / \mathrm{dl}$ & $10.8 \pm 0.8^{b}$ & $9.3 \pm 0.5^{\mathrm{c}}$ & $9.5 \pm 0.6^{\mathrm{e}}$ & $9.6 \pm 0.6^{\mathrm{f}}$ \\
\hline $\mathrm{Pi}, \mathrm{mg} / \mathrm{dl}$ & $6.0 \pm 0.4^{\mathrm{a}}$ & $3.4 \pm 0.6^{\mathrm{d}}$ & $4.9 \pm 0.6^{\mathrm{f}}$ & $5.0 \pm 0.7^{\mathrm{f}}$ \\
\hline Albumin, g/dl & $3.97 \pm 0.41$ & $3.99 \pm 0.39$ & $3.96 \pm 0.36$ & $3.97 \pm 0.37$ \\
\hline
\end{tabular}

Note: ${ }^{\mathrm{a}} P<.05$, ${ }^{\mathrm{b}} P<.01$, compared with Control at D0; $\mathrm{c} P<.05$, $\mathrm{d} P<.01$, compared with D0 (PTX); ${ }^{\mathrm{e}} P<$ $.05,{ }^{\mathrm{f}} P<.01$, compared with PTX group at M12.

Alk-P, alkaline phosphatase; BMD, bone mineral density; D0, Day 0, before PTX; FC BMD, Fractional change (\%) in BMD between D0 and M12; FN, femoral neck; HD, hemodialysis; M12, 12 months after PTX; iPTH, intact parathyroid hormone; LS, lumbar spine; OPG, osteoprotegerin; PTX, parathyroidectomy; Pi, inorganic phosphate; TCa, Total calcium.

Reference value: iPTH, 10-65 pg/ml; Alk-P, 60-170 U/1; OPG, $259.7 \pm 65.2$ pg/ml; TCa, 8.5-10.5 mg/dl; Pi, 2.3-4.6 mg/dl; Albumin, 3.5-4.5 g/dl. 
the baseline OPG level tended to be lower in post-menopausal women than in pre-menopausal women $(1,568.72 \pm$ 541.6 vs $2,389.4 \pm 748.8$ respectively; $P=.053)$. None of the patients showed signs of malnutrition, and all had normal serum albumin levels $(3.97 \pm 0.41 \mathrm{~g} / \mathrm{dl})$.

\section{Baseline BMD}

As shown in Table 1, the baseline (D0) BMD of FN and LS were $0.72 \pm 0.07 \mathrm{~g} / \mathrm{cm}^{2}$ and $0.97 \pm 0.07 \mathrm{~g} / \mathrm{cm}^{2}$, respectively, in patients receiving PTX. For the control group, the D0 BMD of FN and LS were $0.79 \pm 0.08 \mathrm{~g} / \mathrm{cm}^{2}$ and $1.02 \pm 0.08 \mathrm{~g} / \mathrm{cm}^{2}$, respectively. Prior to PTX, the baseline T-scores for FN and LS were significantly lower in patients with SHPT compared to control patients $(P<.05$ in both instances). The FN T-score for the study patients was significantly lower than their LS T-score $(P<.001)$.

We found that the baseline LS T-scores were significantly lower in post-menopausal women compared to the male participants $(-0.76 \pm 0.72$ vs $-0.17 \pm 0.45$ respectively; $P<.05$ ). Furthermore, the baseline LS T-scores tended to be lower in post-menopausal women than in the pre-menopausal women $(-0.76 \pm 0.72$ vs $-0.21 \pm 0.33$; $P=$ $.07)$.

\section{Biochemical parameter changes after PTX}

After PTX, the study patients' serum bone markers improved. No reported permanent hypoparathyroidism occurred. There was a marked fall in iPTH levels after PTX, starting on D2 and continuing thereafter (D0: 1,263.8 \pm 543.0 vs D2: $63.8 \pm 37.1 \mathrm{pg} / \mathrm{ml} ; P<.001$ ) (Fig. 1a). This pattern was sustained through to M12 $(48.1 \pm 19.5 \mathrm{pg} / \mathrm{ml}$ at M12; $P<.001$ compared with D0). The histomorphological changes revealed by bone biopsy are the gold standard for diagnosis of renal osteodystrophy. However, several serum bone markers are more conveniently used to measure changes in bone turnover (Malluche et al. 2011). None of our patients had biochemical hypoparathyroidism, which was defined as iPTH levels $<20 \mathrm{pg} / \mathrm{ml}$ (Puccini et al. 2010) at long-term follow up. However, we could not exclude the possibility of low turnover bone disease because the mean serum intact PTH of our sample was $48.1 \pm 19.5 \mathrm{pg} / \mathrm{ml} 1$ year after PTX (Goodman et al. 1994; Yajima et al. 2001).

A significant progressive increase in serum Alk-P levels commenced on D0 and peaked at Week 2 post-PTX (D0: $610.3 \pm 258.0$ vs $\mathrm{W} 2: 896.5 \pm 268.0 \mathrm{IU} / \mathrm{L} ; P<.001)$. Thereafter, the serum Alk-P level progressively decreased, and had returned to baseline levels by M1 $(639.9 \pm 212.0$ IU/L; $P<.001)$. Nonetheless, M12 serum Alk-P levels were higher in study patients than in controls $(P<.01)$ (Fig. $1 b)$. In contrast, serum OPG levels fell slightly during the immediate post-PTX period and started to increase after 2 weeks (D0: $2,167.1 \pm 767.8$ vs W2: $2,509.4 \pm 611.7 \mathrm{pg} / \mathrm{ml}$ ). Thereafter, OPG levels gradually rose and reached a peak at 2 months post-PTX $(2,882.1 \pm 730.2 \mathrm{pg} / \mathrm{ml})$. After this period, the serum OPG level again fell gradually, but remained above normal levels by the end of the first year
$(2,826.0 \pm 741.5 \mathrm{pg} / \mathrm{ml})$ (Fig. 1c). Both OPG and Alk-P is secreted by osteoblasts; thus, a progressive decrease in osteoblast activity as reflected in serum Alk-P level changes may explain the progressive decrease of serum OPG within 2 months post-PTX.

Temporary hypocalcemia was observed in all patients who received PTX. Serum calcium as measured at D0, D2, $\mathrm{W} 1, \mathrm{~W} 2, \mathrm{M} 1, \mathrm{M} 3, \mathrm{M} 6, \mathrm{M} 9$, and M12 were $10.83 \pm 0.83$, $6.79 \pm 0.35,6.96 \pm 0.53,7.23 \pm 0.61,8.59 \pm 0.58,8.96 \pm$ $0.58,9.05 \pm 0.66,8.89 \pm 0.58$, and $9.24 \pm 0.47 \mathrm{mg} / \mathrm{dl}$, respectively.

\section{BMD changes after PTX}

The T-scores for FN and the LS had increased significantly within 12 months after PTX (for FN, D0 T-score: $-0.94 \pm 0.59$ vs M12 T-score: $-0.39 \pm 0.51 ; P<.001$; for LS, D0 T-score: $-0.28 \pm 0.52$ vs M12 T-score: $0.76 \pm 0.50$; $P<.001$ ) (Table 1). Furthermore, the increase in T-score was significantly greater for LS than for FN $(1.04 \pm 0.27$ vs $0.54 \pm 0.16$ respectively; $P<.001)$. The BMDs of FN and LS measured 1 year after PTX had increased by $8.3 \%$ and $12.3 \%$, respectively.

\section{Correlation analyses}

We calculated univariate Spearman correlation coefficients to determine the relationships between serum iPTH, Alk-P, and OPG levels and changes in FN and LS T-scores at different times after PTX. For FN, baseline iPTH showed a significant and positive correlation with T-score changes $(r=0.78, P<.01)$, with the same pattern being evident for LS $(r=0.80, P<.01)$. Early-month serum Alk-P levels (D0 - M3) correlated significantly with changes in LS T-score 1 year after PTX $(P<.05)$. Although baseline serum OPG level did not correlate significantly with BMD, the change in OPG level between baseline and second month ( $\triangle \mathrm{M} 2-\mathrm{D} 0)$ was positively correlated with lumbar spine T-score changes 1 year after surgery $(r=0.53$, $P<.05)$.

Fig. 2a shows the increment of serum OPG level [peak percentage change: $(\mathrm{M} 2-\mathrm{D} 0) / \mathrm{D} 0 \times 100 \%$ ] was positively associated with baseline iPTH (D0 iPTH) level $(r=0.407$, $P<.05)$. Thus, when pre-PTX levels of PTH were high, post-PTX levels of OPG were also high. In Fig. $2 b$, the increment of serum OPG level was negatively associated with the increment in serum Alk-P level [peak percentage change: (W2-D0)/W2 $\times 100 \%$ level $(r=-0.44, P<.05)$, suggesting elevated bone formation may still be coupled with higher bone resorption after PTX.

\section{Discussion}

This study demonstrated that iPTH levels dramatically decreased following PTX. Serum Alk-P started to increase on postoperative on Day 2, and peaked at 2 weeks postPTX. Serum OPG levels began to increase at postoperative Week 2, and peaked roughly 2 months after PTX. The baseline iPTH level was positively correlated with T-score 
a.

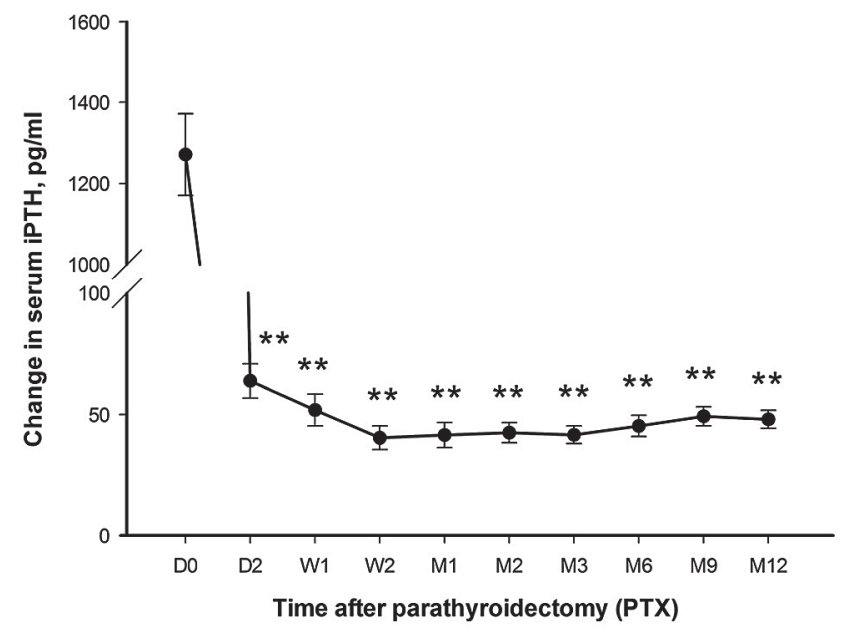

b.

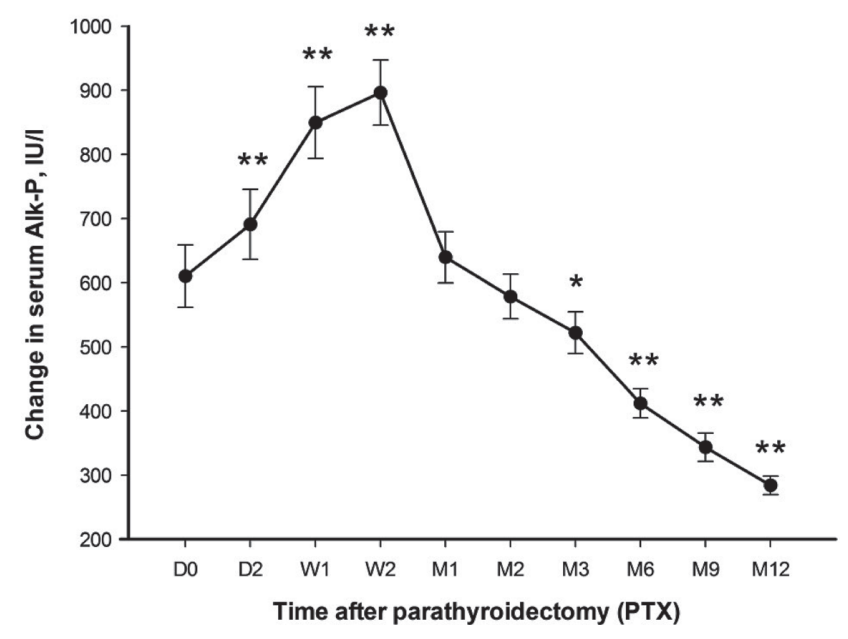

c.

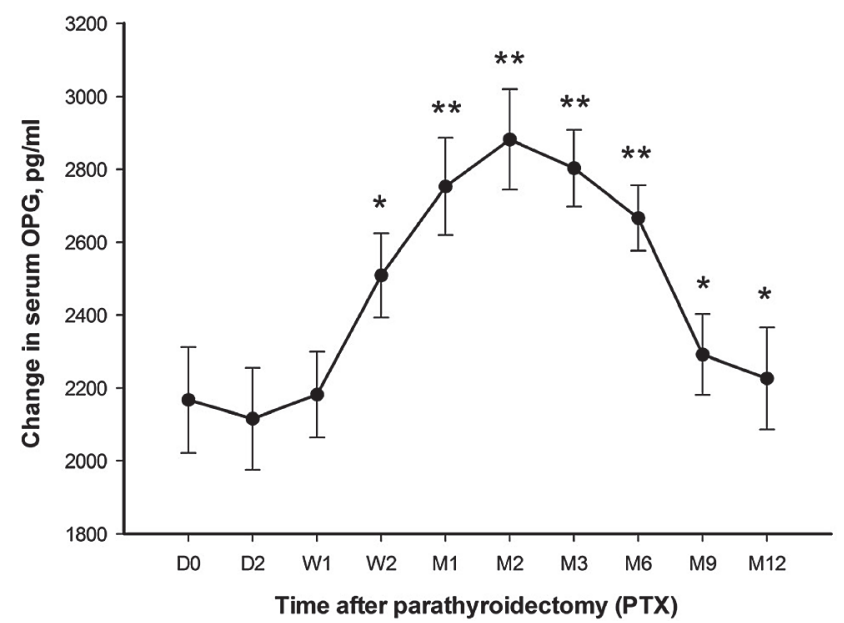

Fig. 1. Serial serum levels of bone metabolism markers before and after parathyroidectomy (PTX). (a) intact parathyroid hormone (iPTH), (b) alkaline phosphatase (Alk-P) and (c) osteoprotegerin (OPG). Before PTX: baseline (D0). After PTX: 1 day later (D2), 1 week (W1), 2 weeks (W2), 1 month (M1), 2 months (M2), 3 months (M3), 6 months (M6), 9 months (M9), and 12 months (M12). Alk-P levels peaked at W2 and OPG levels peaked at M2.

$* P<.05$ and $* * P<.01$ when compared with baseline data (D0). 
a.

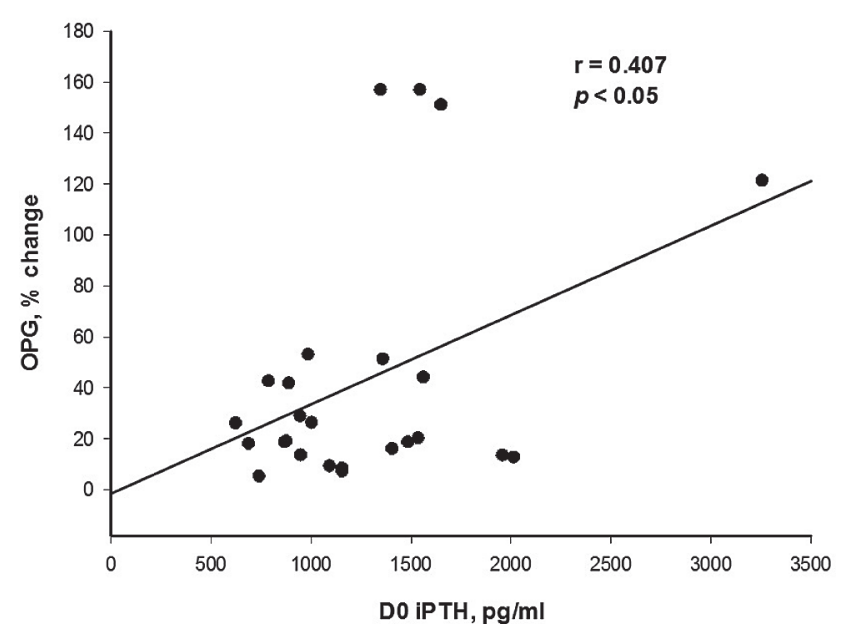

b.

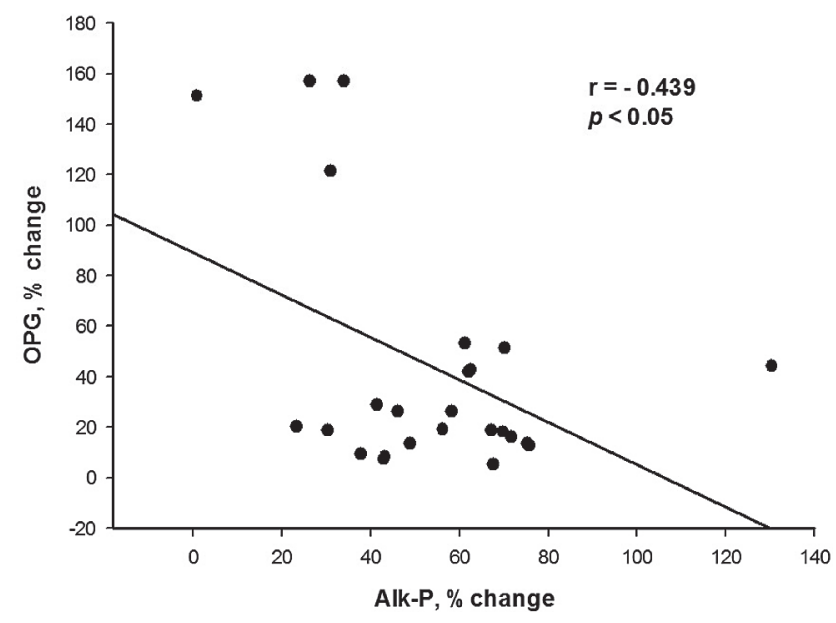

Fig. 2. Relationships between baseline iPTH, increment of serum OPG level and increment in serum Alk-P level. (a) The increment of serum OPG level [peak percentage change: $(\mathrm{M} 2-\mathrm{D} 0) / \mathrm{D} 0 \times 100 \%$ ] was positively associated with baseline iPTH (D0 iPTH) level $(r=0.407, P<.05)$. (b) The increment of serum OPG level was negatively associated with the increment in serum Alk-P level [peak percentage change: (W2-D0)/W2 $\times 100 \%$ ] level $(r=-0.44, P<.05)$.

changes in both FN and LS, as well as with the peak percentage change in OPG. The rise in serum OPG was positively related to increased LS BMD, suggesting that inhibition of osteoclastic bone resorption may play an important role in the BMD improvements within 1 year after PTX.

This increased Alk-P levels observed during the early post-PTX period may be associated with hungry bone syndrome, where rapidly increased deposition of calcium into the bone results in severe hypocalcemia and improved in BMD (Peretz et al. 1992; Franca et al. 2011). In patients with pre-dialysis chronic kidney disease or those on maintenance HD, elevated Alk-P is associated with increased vascular calcification and cardiovascular mortality (Schoppet and Shanahan 2008; Shantouf et al. 2009). Serum Alk-P promotes mineralization by removing inhibitory pyrophosphate (O’Neill 2006), therefore, Alk-P levels could predict a patient's calcium requirement after PTX (Goh et al. 2010). The increase in Alk-P after PTX may be caused by a rise in type 2 osteoblasts (Jilka et al. 1999), but we did not investigate this possibility. The progressive decline in Alk-P concentration after Week 2 post-PTX is probably due to the removal of the persistent PTH stimulus. Post-PTX calcium and calcitriol supplement may also have an impact. However, a previous study suggested that calcitriol usage in the post-PTX period does not play a major role in modulating serum Alk-P levels (Mazzaferro et al. 2000).

Osteoprotegerin (OPG), also known as osteoclastogenesis inhibitory factor (OCIF), or tumor necrosis factor receptor superfamily member 11B (TNFRSF11B), is a protein of the tumor necrosis factor (TNF) receptor superfamily. OPG blocks the interaction between nuclear factor-kB (RANK) and its ligand (RANKL), thus inhibiting osteoclast differentiation and activity, and consequently preventing bone resorption (Bolon et al. 2001). Calciotropic hormones and cytokines, including PTH, apparently act through a 
dual mode to inhibit OPG production and stimulate RANKL production (Vega et al. 2007). Previous in vitro studies of osteoblasts have indicated that PTH either decreases (Huang et al. 2004) or does not affect (Coetzee et al. 2007) OPG levels. Decreased OPG gene expression was detected in bone biopsies of patients with primary hyperparathyroidism (PHPT), and was reversed after PTX (Stilgren et al. 2004). However, serum OPG did not correlate with PTH before surgery and was unaffected by PTX (Stilgren et al. 2003) and this finding may relate to intact kidney excretory function. Another study reported that endogenous PTH levels were negatively associated with OPG expression in human bone tissue (Seck et al. 2001). The present study showed that high pre-PTX levels of PTH were associated with high post-PTX levels of OPG. This suggests that before PTX, high PTH levels may suppress OPG secretion and that PTX may remove the suppressive effects of PTH, resulting in markedly increased serum OPG levels. Our findings further suggested that increased bone formation and decreased bone resorption during the postPTX period were coordinated by Alk-P and OPG; however, this possibility requires further investigation.

Serum OPG concentrations are reduced in low-turnover renal bone disorders, while high PTH levels have been observed in high bone turnover renal osteodystrophy (Coen et al. 2002). The increased serum OPG concentrations in patients with chronic kidney disease may be an adaptive mechanism to attenuate PTH-induced bone loss (Kazama et al. 2002). The sensitivity of PTH to bone differs among dialysis patients (Andress et al. 1987; Slatopolsky et al. 2000; Iwasaki et al. 2006). Thus, the relationship between PTH and OPG may also differ among these patients. Because the severity of high bone turnover depends partly on factors other than PTH, one may conclude that the effects of PTX on BMD and serum OPG also differ among patients. In the present study, the 1-84PTH/7-84PTH ratio was not determined, and dialysis patients with diabetes mellitus were included among the patients treated with PTX. We found that baseline iPTH before PTX was significantly and positively correlated with increased BMD in FN and LS. Thus, a higher pre-PTX level of iPTH indicates a higher BMD at 1 year post-surgery. The increment of serum OPG level was also directly associated with the baseline iPTH level, indicating that when pre-PTX iPTH correlates with high post-PTX OPG, consequently decreasing bone resorption and eventually resulting in increased BMD.

We also found that serum OPG increased progressively until 2 months after PTX. A previous histomorphological study reported that osteoblast surface increased until 1 week after PTX in cancellous bone, but that a progressive increase continued until 4 weeks post-PTX in the cortical bone (Yajima et al. 2007). Because serum OPG is secreted by osteoblasts, these biopsy findings may explain the increased serum OPG levels observed in the early weeks after PTX in our cases. Serum OPG levels progressively decreased between postoperative Month 2 and Month 12, but remained higher than at baseline, suggesting that relatively suppressed bone resorption (through OPG) may persist for at least 12 months after PTX. In addition, the increment in Alk-P level was negatively associated with the increment in OPG level, suggesting that elevated bone formation may still be coupled with higher bone resorption after PTX.

Bone remodeling normally starts with the creation of resorption pits by osteoclasts, followed by apoptosis of osteoclasts, then osteoblast formation and mineralization (Parfitt 1998; Manolagas 2000). However, this does not apply to the PTX patients in our study. The pathophysiology of hungry bone syndrome after PTX is similar to the bone marrow ablation model that has been used to investigate osteogenic capacity (Tanaka et al. 2002). Bone marrow ablation induces the rapid activation of bone synthetic activity. Initially, bone formation markers (e.g., Alk-P) were expressed in the bone formation phase, followed by the stimulation of OPG expression in the bone resorption phase (Tanaka et al. 2011). This finding is in line with our observation that, following PTX, there was a 4- to 8-week lag between changes in Alk-P and the subsequent parallel changes in OPG levels.

Serum OPG is not decreased in patients with PHPT, and its level is unaffected by PTX, despite an obvious reduction in PTH levels (Stilgren et al. 2003). This may be due to the normal renal excretion of OPG that occurs in PHPT patients, unlike in renal failure patients (Stilgren et al. 2004). High PTH and low estradiol inhibit OPG production; in a study of post-menopausal HD patients with moderate hyperparathyroidism, low serum OPG was associated with low BMD (Wu et al. 2010). In our study, both the baseline LS T-scores and serum OPG levels in post-menopausal women tended to be lower than those of pre-menopausal women before PTX; however, these differences were not statistically significant. The small sample size may have affected our statistical results.

Aortic calcification (AC) has a moderating influence on spine BMD measurements. Although overestimation of BMD is generally minimal, severe aortic calcification may distort LS assessments (Frye et al. 1992). Thus, we cannot exclude the influence of AC on LS BMD values. Our study was also limited by its small sample size, non-randomized design, and because bone Alk-P (a more specific enzyme subtype) and RANKL were not measured. Serum OPG may not reflect the levels and activity of these cytokines in the bone microenvironment. Thus, further studies, including histomorphometric analysis, are necessary.

In conclusion, this investigation of serial changes in bone markers revealed that the rapid decrease in serum iPTH levels after PTX was related to suppression of bone resorption and improvement of bone formation. Baseline iPTH was positively correlated with BMD changes in FN and LS, as well as increment in serum OPG levels. In addition, the increased serum OPG was positively related to the increment in LS BMD, suggesting that inhibition of osteo- 
clastic bone resorption may play an important role in improving BMD within the first year after PTX.

\section{Acknowledgments}

This work was supported by a grant from the Tri-Service General Hospital (TSGH-C100-081) for P. Chu and by a grant from the Cardinal-Tien Hospital (CTH-93-1-2A03) for K-C Lu.

\section{Conflict of Interest}

The authors declare no conflict of interest.

\section{References}

Andress, D.L., Hercz, G., Kopp, J.B., Endres, D.B., Norris, K.C., Coburn, J.W. \& Sherrard, D.J. (1987) Bone histomorphometry of renal osteodystrophy in diabetic patients. J. Bone Miner. Res., 2, 525-531.

Bolon, B., Carter, C., Daris, M., Morony, S., Capparelli, C., Hsieh, A., Mao, M., Kostenuik, P., Dunstan, C.R., Lacey, D.L. \& Sheng, J.Z. (2001) Adenoviral delivery of osteoprotegerin ameliorates bone resorption in a mouse ovariectomy model of osteoporosis. Mol. Ther, 3, 197-205.

Caplan, L., Hines, A.E., Williams, E., Prochazka, A.V., Saag, K.G., Cunningham, F. \& Hutt, E. (2011) An observational study of glucocorticoid-induced osteoporosis prophylaxis in a national cohort of male veterans with rheumatoid arthritis. Osteoporos. Int., 22, 305-315.

Chen, H.H., Lin, C.J., Wu, C.J., Lai, C.T., Lin, J., Cheng, S.P. \& Yang, T.L. (2011) Chemical ablation of recurrent and persistent secondary hyperparathyroidism after subtotal parathyroidectomy. Ann. Surg., 253, 786-790.

Coen, G., Ballanti, P., Balducci, A., Calabria, S., Fischer, M.S., Jankovic, L., Manni, M., Morosetti, M., Moscaritolo, E., Sardella, D. \& Bonucci, E. (2002) Serum osteoprotegerin and renal osteodystrophy. Nephrol. Dial. Transplant., 17, 233-238.

Coetzee, M., Haag, M. \& Kruger, M.C. (2007) Effects of arachidonic acid, docosahexaenoic acid, prostaglandin E(2) and parathyroid hormone on osteoprotegerin and RANKL secretion by MC3T3-E1 osteoblast-like cells. J. Nutr. Biochem., 18, 54-63.

Coulston, J.E., Egan, R., Willis, E. \& Morgan, J.D. (2010) Total parathyroidectomy without autotransplantation for renal hyperparathyroidism. Br. J. Surg., 97, 1674-1679.

Doumouchtsis, K.K., Kostakis, A.I., Doumouchtsis, S.K., Tziamalis, M.P., Stathakis, C.P., Diamanti-Kandarakis, E., Dimitroulis, D. \& Perrea, D.N. (2008) Associations between osteoprotegerin and femoral neck BMD in hemodialysis patients. $J$. Bone Miner. Metab., 26, 66-72.

Epstein, S., Dissanayake, I.R., Goodman, G.R., Bowman, A.R., Zhou, H., Ma, Y. \& Jee, W.S. (2001) Effect of the interaction of parathyroid hormone and cyclosporine a on bone mineral metabolism in the rat. Calcif. Tissue Int., 68, 240-247.

Floege, J., Kim, J., Ireland, E., Chazot, C., Drueke, T., de Francisco, A., Kronenberg, F., Marcelli, D., Passlick-Deetjen, J., Schernthaner, G., Fouqueray, B. \& Wheeler, D.C. (2011) Serum iPTH, calcium and phosphate, and the risk of mortality in a European haemodialysis population. Nephrol. Dial. Transplant., 26, 1948-1955.

Franca, T.C., Griz, L., Pinho, J., Diniz, E.T., Andrade, L.D., Lucena, C.S., Beserra, S.R., Asano, N.M., Duarte, A.P. \& Bandeira, F. (2011) Bisphosphonates can reduce bone hunger after parathyroidectomy in patients with primary hyperparathyroidism and osteitis fibrosa cystica. Rev. Bras. Reumatol., 51, 131-137.

Frazao, J.M. \& Martins, P. (2009) Adynamic bone disease: clinical and therapeutic implications. Curr. Opin. Nephrol. Hypertens., 18, 303-307.
Frye, M.A., Melton III, L.J., Bryant, S.C., Fitzpatricka, L.A., Wahner, H.W., Schwartzd, R.S. \& Riggsa, B.L. (1992) Osteoporosis and calcification of the aorta. Bone Miner., 19, 185194.

Goh, B.L., Yudisthra, M.G. \& Hisham, A.N. (2010) Alkaline phosphatase predicts calcium requirements after total parathyroidectomy in patients receiving dialysis. Br. J. Surg., 97, 185-188.

Goodman, W.G., Ramirez, J.A., Belin, T.R., Chon, Y., Gales, B., Segre, G.V. \& Salusky, I.B. (1994) Development of adynamic bone in patients with secondary hyperparathyroidism after intermittent calcitriol therapy. Kidney Int., 46, 1160-1166.

Grzegorzewska, A.E. \& Mlot, M. (2005) Using the ratio of serum osteoprotegerin ligand to osteoprotegerin to evaluate renal osteodystrophy in dialysis patients. Adv. Perit. Dial., 21, 188-193.

Hibi, Y., Tominaga, Y., Sato, T., Katayama, A., Haba, T., Uchida, K., Ichimori, T., Numano, M., Tanaka, Y., Takagi, H., Imai, T., Funahashi, H. \& Nakao, A. (2002) Reoperation for Renal Hyperparathyroidism. World J. Surg., 26, 1301-1307.

Huang, G.S., Chu, T.S., Lou, M.F., Hwang, S.L. \& Yang, R.S. (2009) Factors associated with low bone mass in the hemodialysis patients - a cross-sectional correlation study. B.M.C. Musculoskelet. Disord., 10, 60.

Huang, J.C., Sakata, T., Pfleger, L.L., Bencsik, M., Halloran, B.P., Bikle, D.D. \& Nissenson, R.A. (2004) PTH differentially regulates expression of RANKL and OPG. J. Bone Miner. Res., 19, 235-244.

Iwasaki, Y., Yamato, H., Nii-Kono, T., Fujieda, A., Uchida, M., Hosokawa, A., Motojima, M. \& Fukagawa, M. (2006) Insufficiency of PTH action on bone in uremia. Kidney Int., 102, S34-36.

Jilka, R.L., Weinstein, R.S., Bellido, T., Roberson, P., Parfitt, A.M. \& Manolagas, S.C. (1999) Increased bone formation by prevention of osteoblast apoptosis with parathyroid hormone. J. Clin. Invest., 104, 439-446.

Kazama, J.J., Shigematsu, T., Yano, K., Tsuda, E., Miura, M., Iwasaki, Y., Kawaguchi, Y., Gejyo, F., Kurokawa, K. \& Fukagawa, M. (2002) Increased circulating levels of osteoclastogenesis inhibitory factor (osteoprotegerin) in patients with chronic renal failure. Am. J. Kidney Dis., 39, 525-532.

Malluche, H.H. \& Faugere, M.C. (1990) Effects of 1,25(OH)2D3 administration on bone in patients with renal failure. Kidney Int., 29, S48-53.

Malluche, H.H., Mawad, H.W. \& Monier-Faugere, M.C. (2011) Renal osteodystrophy in the first decade of the new millennium: analysis of 630 bone biopsies in black and white patients. J. Bone Miner. Res., 26, 1368-1376.

Manolagas, S.C. (2000) Birth and death of bone cells: basic regulatory mechanisms and implications for the pathogenesis and treatment of osteoporosis. Endocr. Rev., 21, 115-137.

Mazzaferro, S., Chicca, S., Pasquali, M., Zaraca, F., Ballanti, P., Taggi, F., Coen, G., Cinotti, G.A. \& Carboni, M. (2000) Changes in bone turnover after parathyroidectomy in dialysis patients: role of calcitriol administration. Nephrol. Dial. Transplant., 15, 877-882.

Melamed, M.L., Eustace, J.A., Plantinga, L.C., Jaar, B.G., Fink, N.E., Parekh, R.S., Coresh, J., Yang, Z., Cantor, T. \& Powe, N.R. (2008) Third-generation parathyroid hormone assays and all-cause mortality in incident dialysis patients: the CHOICE study. Nephrol. Dial. Transplant., 23, 1650-1658.

Miller, P.D. (2009) Diagnosis and treatment of osteoporosis in chronic renal disease. Semin. Nephrol., 29, 144-155.

O’Neill, W.C. (2006) Pyrophosphate, alkaline phosphatase, and vascular calcification. Circ. Res., 99, e2.

Parfitt, A.M. (1998) A structural approach to renal bone disease. J. Bone Miner. Res., 13, 1213-1220.

Peretz, A.M., Dhaene, M.M., Drowart, A., Kinnaert, P., Vertongen, F.M. \& Bourdoux, P.P. (1992) Evolution of osteocalcin, alka- 
line phosphatase and parathyroid hormone after parathyroidectomy in patients receiving chronic maintenance hemodialysis. J. Rheumatol., 19, 411-415.

Puccini, M., Carpi, A., Cupisti, A., Caprioli, R., Iacconi, P., Barsotti, M., Buccianti, P., Mechanick, J., Nicolini, A. \& Miccoli, P. (2010) Total parathyroidectomy without autotransplantation for the treatment of secondary hyperparathyroidism associated with chronic kidney disease: clinical and laboratory long-term follow-up. Biomed. Pharmacother, 64, 359-362.

Schoppet, M. \& Shanahan, C.M. (2008) Role for alkaline phosphatase as an inducer of vascular calcification in renal failure? Kidney Int., 73, 989-991.

Seck, T., Diel, I., Bismar, H., Ziegler, R. \& Pfeilschifter, J. (2001) Serum parathyroid hormone, but not menopausal status, is associated with the expression of osteoprotegerin and RANKL mRNA in human bone samples. Eur. J. Endocrinol., 145, 199-205.

Shantouf, R., Kovesdy, C.P., Kim, Y., Ahmadi, N., Luna, A., Luna, C., Rambod, M., Nissenson, A.R., Budoff, M.J. \& KalantarZadeh, K. (2009) Association of serum alkaline phosphatase with coronary artery calcification in maintenance hemodialysis patients. Clin. J. Am. Soc. Nephrol., 4, 1106-1114.

Sherrard, D.J., Hercz, G., Pei, Y., Maloney, N.A., Greenwood, C., Manuel, A., Saiphoo, C., Fenton, S.S. \& Segre, G.V. (1993) The spectrum of bone disease in end-stage renal failure - an evolving disorder. Kidney Int., 43, 436-442.

Slatopolsky, E., Finch, J., Clay, P., Martin, D., Sicard, G., Singer, G., Gao, P., Cantor, T. \& Dusso, A. (2000) A novel mechanism for skeletal resistance in uremia. Kidney Int., 58, 753-761.

Stilgren, L.S., Hegedus, L.M., Beck-Nielsen, H. \& Abrahamsen, B. (2003) Osteoprotegerin levels in primary hyperparathyroidism: effect of parathyroidectomy and association with bone metabolism. Calcif. Tissue Int., 73, 210-216.

Stilgren, L.S., Rettmer, E., Eriksen, E.F., Hegedus, L., BeckNielsen, H. \& Abrahamsen, B. (2004) Skeletal changes in osteoprotegerin and receptor activator of nuclear factorkappab ligand mRNA levels in primary hyperparathyroidism: effect of parathyroidectomy and association with bone metabolism. Bone, 35, 256-265.

Tanaka, H., Mine, T., Ogasa, H., Taguchi, T. \& Liang, CT. (2011) Expression of RANKL/OPG during bone remodeling in vivo. Biochem. Biophys. Res. Commun., 411, 690-694.

Tanaka, H., Wakisaka, A., Ogasa, H., Kawai, S. \& Liang, C.T. (2002) Effect of IGF-I and PDGF administered in vivo on the expression of osteoblast-related genes in old rats. J. Endocrinol., 174, 63-70.

Tentori, F., Blayney, M.J., Albert, J.M., Gillespie, B.W., Kerr, P.G., Bommer, J., Young, E.W., Akizawa, T., Akiba, T., Pisoni, R.L., Robinson, B.M. \& Port, F.K. (2008) Mortality risk for dialysis patients with different levels of serum calcium, phosphorus, and PTH: the Dialysis Outcomes and Practice Patterns Study (DOPPS). Am. J. Kidney Dis., 52, 519-530.

Vega, D., Maalouf, N.M. \& Sakhaee, K. (2007) CLINICAL Review \#: the role of receptor activator of nuclear factorkappaB (RANK)/RANK ligand/osteoprotegerin: clinical implications. J. Clin. Endocrinol. Metab., 92, 4514-4521.

Wu, W.T., Lee, R.P., Wang, C.H., Fang, T.C., Lin, N.T., Chen, I.H. \& Hsu, B.G. (2010) The association of serum osteoprotegerin and osteoporosis in postmenopausal hemodialysis patients: a pilot study. J. Womens Health, 19, 785-790.

Yajima, A., Inaba, M., Ogawa, Y., Tominaga, Y., Tanizawa, T., Inou, T. \& Otsubo, O. (2007) Significance of time-course changes of serum bone markers after parathyroidectomy in patients with uraemic hyperparathyroidism. Nephrol. Dial. Transplant., 22, 1645-1657.

Yajima, A., Inaba, M., Tominaga, Y., Nishizawa, Y., Ikeda, K. \& Ito, A. (2010) Increased osteocyte death and mineralization inside bone after parathyroidectomy in patients with secondary hyperparathyroidism. J. Bone Miner. Res., 25, 2374-2381.

Yajima, A., Ogawa, Y., Ikehara, A., Tominaga, T., Inou, T. \& Otsubo, O. (2001) Development of low-turnover bone diseases after parathyroidectomy and autotransplantation. Int. J. Urol., 8, S76-79.

Yajima, A., Ogawa, Y., Takahashi, H.E., Tominaga, Y., Inou, T. \& Otsubo, O. (2003) Changes of bone remodeling immediately after parathyroidectomy for secondary hyperparathyroidism. Am. J. Kidney Dis., 42, 729-738.

Yano, K., Tsuda, E., Washida, N., Kobayashi, F., Goto, M., Harada, A., Ikeda, K., Higashio, K. \& Yamada, Y. (1999) Immunological characterization of circulating osteoprotegerin/osteoclastogenesis inhibitory factor: increased serum concentrations in postmenopausal women with osteoporosis. J. Bone Miner. Res., 14, 518-527.

Yao, Y., Wang, G., Wang, Z., Wang, C., Zhang, H. \& Liu, C. (2011) Synergistic enhancement of new bone formation by recombinant human bone morphogenetic protein-2 and osteoprotegerin in trans-sutural distraction osteogenesis: a pilot study in dogs. J. Oral. Maxillofac. Surg., 69, e446-455. 